\title{
Holographic renormalization in teleparallel gravity
}

\author{
Martin Krššák ${ }^{\mathrm{a}}$ \\ Instituto de Física Teórica, Universidade Estadual Paulista, R. Dr. Bento Teobaldo Ferraz, 271, Barra Funda, São Paulo, SP 01140-070, Brazil
}

Received: 12 December 2016 / Accepted: 11 January 2017 / Published online: 24 January 2017

(C) The Author(s) 2017. This article is published with open access at Springerlink.com

\begin{abstract}
We consider the problem of IR divergences of the action in the covariant formulation of teleparallel gravity in asymptotically Minkowski spacetimes. We show that divergences are caused by inertial effects and can be removed by adding an appropriate surface term, leading to the renormalized action. This process can be viewed as a teleparallel ana$\log$ of holographic renormalization. Moreover, we explore the variational problem in teleparallel gravity and explain how the variation with respect to the spin connection should be performed.
\end{abstract}

\section{Introduction}

The variational principle plays a central role in theoretical physics. One of the necessary requirements for its consistency is the finiteness of the action. In general relativity, this is known to be a problem due to the presence of IR divergences, and is addressed using either the "background subtraction" method [1] or holographic renormalization [2,3]. In the latter one, IR divergences are cured by adding appropriate surface terms to the action, which in the original case of the asymptotically AdS spacetimes, could be interpreted as counterterms of a dual field theory through the AdS/CFT correspondence [4]. However, later this method turned out to work in other spacetimes that are not known to possess exact holographic duals [5-8], suggesting that the physical mechanism behind counterterms must be related to some universal property of gravity, rather than to some specific realization of the holographic principle. An interesting work in this direction is [9], where the link with topological invariants was proposed.

In this paper, we analyze the problem of IR divergences in the framework of teleparallel gravity, which is a well-

\footnotetext{
a e-mail: krssak@ift.unesp.br
}

known alternative formulation of general relativity that originates with Einstein's unsuccessful attempt to find a unified field theory [10,11]. Later [12-15], it was revived as a gravity theory only, without any unification purposes, that turned out to be equivalent to general relativity. We call here this original approach — for a recent review, see [16] as pure tetrad teleparallel gravity, since the only variable is the tetrad, while the spin connection is assumed to vanish identically in all frames. As a consequence, such theory has a preferred frame and local Lorentz invariance is violated.

Recently, it was discovered that these problems can be avoided if a non-trivial teleparallel spin connection satisfying the vanishing curvature condition is considered. Use of such a connection leads to the covariant teleparallel gravity that respects local Lorentz symmetry. This covariant formulation of teleparallel gravity was first pioneered in [17] in the framework of metric-affine theories [18], and later in [19,20]. For a textbook overview, see [21].

The covariant formulation was fully developed in [22], where it was shown that there exists a certain physically preferred way to choose a spin connection, which is naturally associated with the tetrad, and a method to extract this connection was proposed. The use of this spin connection removes the inertial effects and a finite purely gravitational action is obtained. Therefore, finding the appropriate spin connection can be understood as a renormalization process, where we remove the spurious inertial effects from the action.

In this paper, we build upon the results of [22], and show that the inertial effects represented by the spin connection enter the teleparallel action only through a surface term. Therefore, the action is renormalized by adding an appropriate surface term, which can be viewed as an analog of holographic renormalization in teleparallel gravity. Moreover, based on this construction, we show how to perform the variation with respect to both the tetrad and the spin connection. 


\section{The covariant formulation of teleparallel gravity}

Teleparallel gravity is formulated using tetrad formalism, where the fundamental variable is the tetrad $h_{\mu}^{a},{ }^{1}$ related to the spacetime metric by

$g_{\mu \nu}=\eta_{a b} h_{\mu}^{a} h_{\nu}^{b}$

In order to be able to parallel transport tensors on manifold, we can define various spin connections; each characterized by its curvature and torsion. In general relativity, we use the torsionless Levi-Civita spin connection $\stackrel{\circ}{\omega}_{b \mu}^{a}$, which turns out to be completely determined from a tetrad, and the non-trivial geometry becomes characterized by the curvature tensor. The physical meaning of the components of the Levi-Civita connection can be seen from the geodesic equation

$\frac{\mathrm{d} u^{a}}{\mathrm{~d} s}+\stackrel{\circ}{\omega}_{b \mu}^{a} u^{b} u^{\mu}=0$,

where the spin connection plays a role of the total acceleration, which is due to both gravitational and inertial effects. The presence of the latter can easily be seen from the fact that the components of the spin connection may be non-vanishing in the absence of gravity and change under local Lorentz transformations. The curvature tensor is constructed from derivatives of the connection and plays the physical role of the tidal tensor that is of purely gravitational origin.

In teleparallel gravity, we use a different connection defined by the condition of vanishing curvature

$$
\begin{aligned}
R_{b \mu \nu}^{a}\left(\omega_{b \mu}^{a}\right)= & \partial_{\mu} \omega^{a}{ }_{b \nu}-\partial_{\nu} \omega^{a}{ }_{b \mu} \\
& +\omega^{a}{ }_{c \mu} \omega^{c}{ }_{b \nu}-\omega^{a}{ }_{c \nu} \omega^{c}{ }_{b \mu} \equiv 0,
\end{aligned}
$$

which is satisfied by a pure gauge-like connection [21]

$\omega_{b \mu}^{a}=\Lambda_{c}^{a} \partial_{\mu} \Lambda_{b}^{c}$,

which is completely determined by the Lorentz matrix, and hence is related to inertial effects only. It is sometimes referred to as the purely inertial connection [21].

The teleparallel connection (4) has non-vanishing torsion,

$T_{\mu \nu}^{a}\left(h_{\mu}^{a}, \omega_{b \mu}^{a}\right)=\partial_{\mu} h_{\nu}^{a}-\partial_{\nu} h_{\mu}^{a}+\omega_{b \mu}^{a} h_{\nu}^{b}-\omega_{b \nu}^{a} h_{\mu}^{b}$,

and can be related to the Levi-Civita connection using Ricci theorem [23]

$\omega_{b \mu}^{a}=\stackrel{\circ}{\omega}_{b \mu}^{a}+K_{b \mu}^{a}$,

\footnotetext{
1 We use the convention, where the Latin indices $a, b, \ldots$ run over the tangent space, while the Greek indices $\mu, v, \ldots$ run over spacetime coordinates. The spacetime indices are raised/lowered using the spacetime metric $g_{\mu \nu}$, while the tangent indices using the Minkowski metric $\eta_{a b}$ of the tangent space.
}

where

$K_{b \mu}^{a}=\frac{1}{2}\left(T_{\mu b}^{a}+T_{b \mu}^{a}-T_{b \mu}^{a}\right)$

is the contortion tensor.

The Lagrangian density of teleparallel gravity is given by

$\mathcal{L}=\frac{h}{4 \kappa} T_{\mu \nu}^{a} S_{a}^{\mu \nu}$

where $h=\operatorname{det} h^{a}{ }_{\mu}, \kappa=8 \pi G$ is the gravitational constant (in $c=1$ units), and $S_{a}^{\mu v}$ is the superpotential defined by

$S_{a}^{\mu \nu}=K_{a}^{\mu \nu}-h_{a}^{v} T^{\mu}+h_{a}^{\mu} T^{v}$,

where $T^{\mu}=T_{v}^{v \mu}$ is the vector torsion. The vacuum field equations are obtained by varying (8) with respect to the tetrad, and is given by

$h^{-1} \partial_{\sigma}\left(h S_{a}{ }^{\rho \sigma}\right)-h_{a}^{\mu} S_{b}^{\nu \rho} T_{v \mu}^{b}+\frac{h_{a}^{\rho}}{4} T_{\mu \nu}^{b} S_{b}^{\mu \nu}-\omega_{a \sigma}^{b} S_{b}^{\rho \sigma}=0$.

The variation with respect to the spin connection vanishes identically. We postpone a proof of this statement to the following section, where the central result of this paper, represented by Eq. (19), is derived.

The equivalence of teleparallel gravity and general relativity follows from a straightforward calculation using the Ricci theorem (6), which shows that the Lagrangian (8) is equivalent (up to a surface term) to the Einstein-Hilbert Lagrangian,

$\mathcal{L}=\stackrel{\circ}{\mathcal{L}}_{\mathrm{EH}}-\partial_{\mu}\left(\frac{h}{\kappa} T^{\mu}\right)$,

implying the equivalence of the field equations of teleparallel gravity (10) and general relativity.

To understand the physical meaning of the teleparallel spin connection, let us recall Ricci theorem (6) and interpret it as a decomposition of the Levi-Civita connection into the teleparallel spin connection and the contortion tensor. Obviously, this decomposition is not unique since there are many possible ways to choose the observer (represented by some $\Lambda_{b}^{a}$ ), each corresponding to a different spin connection of the form (4). For example, in the pure tetrad teleparallel gravity [16], where the teleparallel spin connection vanishes identically in all frames, the torsion tensor

$T_{\mu \nu}^{a}\left(h_{\mu}^{a}, 0\right)$

plays the role of the total acceleration and represents both gravity and inertia.

However, in the covariant formulation of teleparallel gravity with the non-trivial connection (4), it turns out to be 
possible to split the Levi-Civita connection in a physically desirable way, where the teleparallel connection represents the inertial effects and the contortion tensor represents gravity only. The self-consistent procedure to perform this split was proposed in [22], which runs according to the following scheme. We start with a tetrad $h_{\mu}^{a}$ obtained as a solution to the field equation with the zero spin connection, and define the reference tetrad $h_{(\mathrm{r})}{ }_{\mu}^{a}$ by setting some parameter that controls the strength of gravity to zero. In practice, we can either choose the gravitational constant to vanish

$\left.h_{(\mathrm{r}) \mu}^{a} \equiv h_{\mu}^{a}\right|_{G \rightarrow 0}$.

or consider the asymptotic limit $r \rightarrow \infty$, where gravity is expected to vanish in the case of the asymptotically Minkowski spacetimes.

Since we want torsion to represent gravity only, we require the torsion to vanish in its absence

$T_{\mu \nu}^{a}\left(h_{(\mathrm{r})}^{a}, \omega_{b \mu}^{a}\right) \equiv 0$,

which completely determines the spin connection in terms of the reference tetrad (13) as

$\omega_{b \mu}^{a}=\stackrel{\circ}{\omega}_{b \mu}\left(h_{(\mathrm{r})}\right)$.

Then the torsion tensor constructed out of the "full" tetrad and the spin connection

$T_{\mu \nu}^{a}\left(h_{\mu}^{a}, \omega_{b \mu}^{a}\right)$

represents purely gravitational torsion with the spurious inertial contributions removed [22].

To elaborate on the choice of the reference tetrad we point out that the reference tetrad is related to the Minkowski spacetime through Eq. (1). Therefore, various reference tetrads represent different physical observers in the Minkowski spacetime. There exists a simple diagonal tetrad in the Cartesian coordinate system for which the connection (15) vanishes identically. All the other reference tetrads are related to it by a coordinate change and/or a local Lorentz transformation. If they are related by a local Lorentz transformation, a non-trivial spin connection is generated on the account of non-tensorial transformation properties of the spin connection under local Lorentz transformations. This insight can be used to determine the proper reference tetrad in the case when the limit procedure (13) is not easily achievable.

\section{Surface terms in the teleparallel action}

We expect the action constructed from the torsion tensor with vanishing spin connection (12) to be IR divergent due to the presence of the inertial effects. The inertial forces are just fictitious forces and, unlike the "real" forces associated with physical fields, do not necessarily vanish at infinity [24]. Since the action $S\left(h_{\mu}^{a}, 0\right)$ is an integral of such spurious contributions over the whole spacetime, it is natural to expect it to be IR divergent.

On the other hand, if the action is constructed from the purely gravitational torsion (16), with the spurious inertial contributions removed using the appropriate spin connection (15), we obtain the purely gravitational action $S\left(h_{\mu}^{a}, \omega_{b \mu}^{a}\right)$ that we expect to be IR finite.

The primary task of this paper is to find an explicit relation between these actions. ${ }^{2}$ In principle, it should be possible to achieve this by a straightforward calculation: writing both actions in terms of tetrad and spin connection explicitly, isolating the contributions of the spin connection, and using the zero curvature condition (3), which introduces teleparallelism and relates terms squared in connection to terms linear in their derivatives.

However, it turns out to be possible to achieve the same result in a simpler way, using the equivalence of the teleparallel and the Einstein-Hilbert actions (11). Since the LeviCivita connection of general relativity is a function of a tetrad, the Einstein-Hilbert action can be expressed in terms of a tetrad only. Therefore, the same Einstein-Hilbert action corresponds to both $S\left(h_{\mu}^{a}, 0\right)$ and $S\left(h_{\mu}^{a}, \omega_{b \mu}^{a}\right)$, and we can write

$$
\begin{gathered}
\mathcal{L}\left(h_{\mu}^{a}, \omega_{b \mu}^{a}\right)+\partial_{\mu}\left[\frac{h}{\kappa} T^{\mu}\left(h_{\mu}^{a}, \omega_{b \mu}^{a}\right)\right] \\
=\mathcal{L}\left(h_{\mu}^{a}, 0\right)+\partial_{\mu}\left[\frac{h}{\kappa} T^{\mu}\left(h_{\mu}^{a}, 0\right)\right] .
\end{gathered}
$$

Taking the definition of the torsion tensor (5), and contracting it with $h_{a}^{v}$, we find

$$
\begin{aligned}
T_{\mu}\left(h_{\mu}^{a}, \omega_{b \mu}^{a}\right) & =h_{a}^{\nu} \partial_{\mu} h_{\nu}^{a}-h_{a}{ }^{\nu} \partial_{\nu} h_{\mu}^{a}-h_{a}{ }^{\nu} \omega_{b \nu}^{a} h_{\mu}^{b} \\
& =T_{\mu}\left(h_{\mu}^{a}, 0\right)-\omega_{\mu},
\end{aligned}
$$

where we have defined $\omega_{\mu}=\omega_{b \nu}^{a} h_{a}{ }^{\nu} h_{\mu}^{b}$. Combining these two relations, we find that the Lagrangians are related by a total divergence only,

$\mathcal{L}\left(h_{\mu}^{a}, \omega_{b \mu}^{a}\right)=\mathcal{L}\left(h_{\mu}^{a}, 0\right)+\frac{1}{\kappa} \partial_{\mu}\left(h \omega^{\mu}\right)$

This "holographic" relation is the central result of this paper. It shows that the divergences are removed from the action by adding an appropriate surface term to the action, analogously to holographic renormalization. However, in telepar-

\footnotetext{
${ }^{2}$ We should recall here that there exists a class of frames called proper frames, in which the spin connection vanishes, and these actions coincide [22]. See [25] for an explicit example. Our task here is to derive the relation between actions in the case of the non-vanishing spin connection.
} 
allel gravity it can be interpreted as a removal of the spurious inertial effects from the theory.

This relation allows us to address the variational problem in the covariant teleparallel gravity properly. Since the Lagrangian (8) is a function of both the tetrad and the spin connection, the variation with respect to both variables should be considered. The variation with respect to the tetrad leads straightforwardly to the field equations (10), but the variation with respect to the spin connection is a more tricky task. To illustrate this problem, let us naively consider a straightforward variation of the Lagrangian (8) with respect to the spin connection. We can observe that the spin connection appears in the action without derivatives, and it is easy to find out that the variation leads to $S_{a}^{\mu \nu}=0$, implying a trivial theory. The reason for this puzzling result is that we are naively varying a Lagrangian quadratic in a general torsion, not the teleparallel one.

In order to obtain the correct result, we should keep in mind that the theory with the Lagrangian (8) is a teleparallel theory if and only if we use the teleparallel condition (3), solved by the connection (4). The teleparallel condition (3) relates the terms quadratic in the connection to their derivatives, which turn out to form a total derivative, leading to Eq. (19). Since the spin connection enters the action only through the surface term, the variation with respect to the spin connection vanishes

$\frac{\delta \mathcal{L}\left(h_{\mu}^{a}, \omega_{b \mu}^{a}\right)}{\delta \omega^{a}{ }_{b \mu}}=0$.

Moreover, it implies that the field equations (10) derived from $\mathcal{L}\left(h_{\mu}^{a}, \omega^{a}{ }_{b \mu}\right)$ and $\mathcal{L}\left(h_{\mu}^{a}, 0\right)$ are the same, which allows us to solve the field equations with the vanishing spin connection. This is crucial for the procedure of determining the spin connection proposed in [22], since the solution is needed to define the reference tetrad (13), and consequently the correct spin connection (15). Furthermore, this result explains why in pure tetrad teleparallel gravity the solutions to the field equations are correct [12-16]. It illustrates actually the irrelevance of the teleparallel spin connection for obtaining the solution of the field equations.

\section{Schwarzschild solution}

To illustrate the renormalization process, let us consider the diagonal tetrad representing the Schwarzschild solution in the spherical coordinate system

$h_{\mu}^{a}=\operatorname{diag}(\sqrt{f(r)}, 1 / \sqrt{f(r)}, r, r \sin \theta)$,

where

$f(r)=1-2 G M / r$.
The unrenormalized Lagrangian density is given by

$\mathcal{L}\left(h_{\mu}^{a}, 0\right)=\frac{1}{\kappa} \sin \theta$.

The non-vanishing components of the spin connection corresponding to the diagonal tetrad (21) are

$\omega_{\hat{2} \theta}^{\hat{1}}=-1, \quad \omega_{\hat{3} \phi}^{\hat{1}}=-\sin \theta, \quad \omega_{\hat{3} \phi}^{\hat{2}}=-\cos \theta$.

We find the contraction

$\omega^{\mu}=\left(0,-\frac{2 f}{r},-\frac{\cot \theta}{r^{2}}, 0\right)$,

and we construct the surface term

$\partial_{\mu}\left(h \omega^{\mu}\right)=\left[1+2 \frac{(G M-r)}{f r}\right] \sin \theta$.

We can check that adding this surface term to the unrenormalized action (23) according to Eq. (19) leads to the renormalized Lagrangian

$\mathcal{L}\left(h^{a}{ }_{\mu}, \omega^{a}{ }_{b \mu}\right)=\frac{2}{\kappa}\left[1+\frac{(G M-r)}{r \sqrt{f}}\right] \sin \theta$,

and the renormalized action [22].

\section{Conclusions}

In this paper we have analyzed the problem of IR divergences of the action in the covariant formulation of teleparallel gravity in asymptotically Minkowski spacetimes. We have shown that, since torsion is related to the acceleration of the observer, there is a possible mixing of gravitational and inertial effects. The inertial effects are not related to the actual physical fields, and hence do not necessarily vanish at infinity, which leads to IR divergences of the teleparallel action. It is an important physical insight provided by teleparallel gravity that the IR divergences of the action are actually physically meaningful and are related to inertial effects.

These spurious inertial contributions can be removed, provided the appropriately chosen teleparallel spin connection (representing the inertial effects in the tetrad) is used. We have then analyzed how this spin connection enters the teleparallel action, which led us to the "holographic" relation (19) that shows that the spin connection enter the action only through the surface term. Therefore, the removal of the spurious inertial contributions can be understood as an addition of the appropriate surface term that renders the action finite, which can be considered as a teleparallel analog of holographic renormalization. 
An interesting aspect of renormalization of the teleparallel action is that it has features of both holographic renormalization and background subtraction methods. The analogy with background subtraction was already suggested in [22], where it was observed that the spin connection is calculated from the reference tetrad, representing the reference or background spacetime. The current work provides further insight in that, for the purpose of renormalization of the action, the reference tetrad does not have to be defined everywhere. Since it enters the action only through the surface term, it is sufficient if the reference tetrad is properly defined at infinity.

Equation (19) not only explains how the action is renormalized, but is of crucial importance for the consistency of the variational principle in the covariant formulation of teleparallel gravity. Since the spin connection is a function of the reference tetrad only, it should be considered as an independent variable. Therefore, the variation of the action with respect to the spin connection should be considered as well. The relation (19) allows us to perform this variation easily, to find out that this variation vanishes and the field equations are independent of the choice of the spin connection. This result explains the success of the previous, non-covariant, pure tetrad formulations in finding solutions to teleparallel gravity, and it clarifies the relation with the covariant formulation considered in this paper.

Acknowledgements The author would like to thank José Geraldo Pereira for a fruitful discussion and comments on this manuscript. This work was financially supported by FAPESP (Grant No. 13/10585-6).

Open Access This article is distributed under the terms of the Creative Commons Attribution 4.0 International License (http://creativecomm ons.org/licenses/by/4.0/), which permits unrestricted use, distribution, and reproduction in any medium, provided you give appropriate credit to the original author(s) and the source, provide a link to the Creative Commons license, and indicate if changes were made.

Funded by SCOAP ${ }^{3}$.

\section{References}

1. G.W. Gibbons, S.W. Hawking, Action integrals and partition functions in quantum gravity. Phys. Rev. D 15, 2752 (1977)

2. V. Balasubramanian, P. Kraus, A stress tensor for antide Sitter gravity. Commun. Math. Phys. 208, 413 (1999). arXiv:hep-th/9902121

3. K. Skenderis, Lecture notes on holographic renormalization. Class. Quant. Grav. 19, 5849 (2002). arXiv:hep-th/0209067

4. J.M. Maldacena, The Large N limit of superconformal field theories and supergravity. Adv. Theor. Math. Phys. 2, 231 (1998). arXiv:hep-th/9711200
5. P. Kraus, F. Larsen, R. Siebelink, The gravitational action in asymptotically AdS and flat space-times. Nucl. Phys. B 563, 259 (1999). arXiv:hep-th/9906127

6. V. Balasubramanian, J. de Boer, D. Minic, Mass, entropy and holography in asymptotically de Sitter spaces. Phys. Rev. D 65, 123508 (2002). arXiv:hep-th/0110108

7. F. Larsen, R. McNees, Holography, diffeomorphisms, and scaling violations in the CMB. JHEP 0407, 062 (2004). arXiv:hep-th/0402050

8. R.B. Mann, D. Marolf, Holographic renormalization of asymptotically flat spacetimes. Class. Quant. Grav. 23, 2927 (2006). arXiv:hep-th/0511096

9. O. Miskovic, R. Olea, Topological regularization and self-duality in four-dimensional anti-de Sitter gravity. Phys. Rev. D 79, 124020 (2009). arXiv:0902.2082 [hep-th]

10. A. Einstein, Auf die Riemann-Metrik und den Fern-Parallelismus gegründete einheitliche Feldtheorie. Math. Ann. 102, 685 (1930) arXiv:physics/0503046 (For an English translation together with other related papers, see A. Unzicker, T. Case, "Unified field theory based on Riemannian metrics and distant parallelism)

11. T. Sauer, Field equations in teleparallel spacetime: Einstein's Fernparallelismus approach towards unified field theory. Hist. Math. 33, 399-439 (2006). arXiv:physics/0405142

12. C. Møller, Conservation laws and absolute parallelism in general relativity. K. Dan. Vidensk. Selsk. Mat. Fys. Skr. 1, 10 (1961)

13. K. Hayashi, T. Nakano, Extended translation invariance and associated gauge fields. Prog. Theor. Phys. 38, 491 (1967)

14. Y.M. Cho, Einstein Lagrangian as the translational Yang-Mills Lagrangian. Phys. Rev. D 14, 2521 (1976)

15. K. Hayashi, The gauge theory of the translation group and underlying geometry. Phys. Lett. B 69, 441 (1977)

16. J.W. Maluf, The teleparallel equivalent of general relativity. Ann. Phys. 525, 339 (2013). arXiv:1303.3897 [gr-qc]

17. Y.N. Obukhov, J.G. Pereira, Metric affine approach to teleparallel gravity. Phys. Rev. D 67, 044016 (2003). arXiv:gr-qc/0212080

18. F.W. Hehl, J.D. McCrea, E.W. Mielke, Y. Ne'eman, Metric affine gauge theory of gravity: field equations, Noether identities, world spinors, and breaking of dilation invariance. Phys. Rept. 258, 1 (1995). arXiv:gr-qc/9402012

19. Y.N. Obukhov, G.F. Rubilar, Covariance properties and regularization of conserved currents in tetrad gravity. Phys. Rev. D 73, 124017 (2006). arXiv:gr-qc/0605045

20. T.G. Lucas, Y.N. Obukhov, J.G. Pereira, Regularizing role of teleparallelism. Phys. Rev. D 80, 064043 (2009). arXiv:0909.2418

21. R. Aldrovandi, J.G. Pereira, Teleparallel gravity: an introduction (Springer, Dordrecht, 2012)

22. M. Krššák, J.G. Pereira, Spin connection and renormalization of teleparallel action. Eur. Phys. J. C 75, 1-8 (2015). arXiv: 1504.07683 [gr-qc]

23. M. Nakahara, Geometry, Topology and Physics (Taylor \& Francis, London, 2003)

24. L.D. Landau, E.M. Lifschitz, The classical theory of fields (Pergamon Press, Oxford, 1971)

25. M. Krššák, E. N. Saridakis, The covariant formulation of $\mathrm{f}(\mathrm{T})$ gravity. Class. Quant. Grav. 33(11), 115009 (2016). arXiv:1510.08432 [gr-qc] 